\title{
Am Puls der Zeit
}

Liebe Leserin, lieber Leser,

Vor 70 Jahren, 1946, gründeten die Brüder Dr. Wilhelm Schaeffler und Dr. Georg Schaeffler INA in Herzogenaurach. Seither hat sich das Unternehmen ständig weiterentwickelt, vom Mittelständler zum Weltkonzern, vom Komponentenhersteller zum Systemzulieferer mit Integrationskompetenz.

Der nachhaltige Erfolg des Unternehmens basiert maßgeblich auf der ausgeprägten Innovationskraft und der hohen Fertigungskompetenz, mit der Schaeffler Produkte von der ersten Konzeptidee über den Entwicklungsprozess zur Serienreife bringt. Als Beispiele seien hier nur das revolutionäre käfiggeführte Nadellager, die Tellerfederkupplung von LuK und ganz aktuell der elektromechanische Wankstabilisator genannt. Dieser schlägt gleich zwei Brücken zu Zukunftsthemen. Einerseits ist er ein wichtiger Baustein der Produktstrategie von Schaeffler auf dem Feld der Fahrerassistenz und des (teil)automatisierten Fahrens, andererseits lässt er sich als 48-V-Lösung perfekt mit NiedervoltHybridarchitekturen verknüpfen. Diese bieten das Potenzial, der Kristallisationspunkt für eine weitere Marktverbreitung von elektrifizierten Antriebssystemen zu sein, für die Schaeffler seit einiger Zeit erheblich in Forschung und Entwicklung investiert.

Betrachtet man die Innovationen von Schaeffler der vergangenen 70 Jahre, erkennt man sehr schnell einen weiteren Erfolgsfaktor des Unternehmens: die sehr konsequent am Markt ausgerichtete Produktentwicklung. Das macht die Innovationen von Schaeffler zu einem Spiegelbild der Automobilentwicklung, an dem sich exemplarisch der „Puls der Zeit“ der jeweiligen Dekade ablesen lässt - vom Wirtschaftwunder der Gründungsjahre über Ölkrise, Umweltschutz und Dieselboom bis hin zur Elektrifizierung des Antriebs mit Hybrid- und reinen E-Konzepten.

Steigen Sie ein und begleiten Sie uns auf unserer automobilen Zeitreise in die Vergangenheit und in die Zukunft, mit historischen Rückblicken und klaren Perspektiven für die Mobilität von morgen.

Viel Spaß bei der Lektüre dieses gemeinsam mit Schaeffler erstellten ATZextra wünscht Ihnen

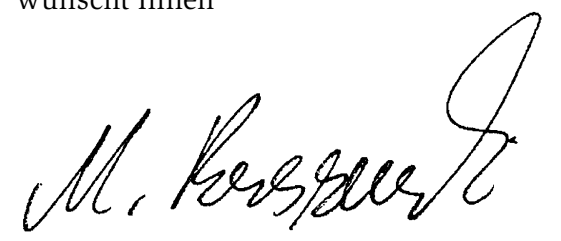

Markus Bereszewski, Leitender Redakteur Sonderprojekte und internationale Medien

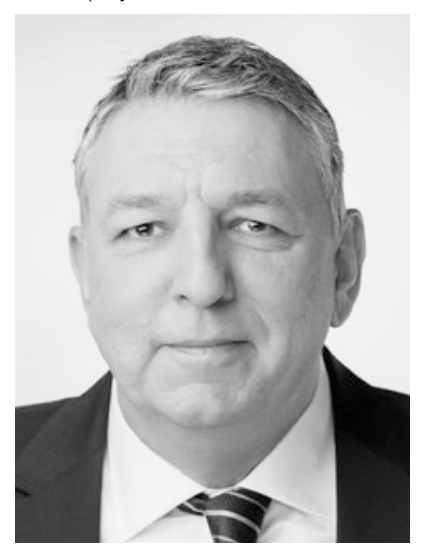

